\section{Determinants of quality of life at first presentation} with schizophrenia

\author{
STEPHEN BROWNE, MARY CLARKE, MAURICE GERVIN, \\ JOHN L. WADDINGTON, CONAL LARKIN and EADBHARD O'CALLAGHAN
}

\author{
Background Quality of life (QOL) has \\ gained importance as a global measure of \\ social and clinical outcome in \\ schizophrenia.
}

\begin{abstract}
Aims To identify the clinical correlates of QOL at the time of first presentation with schizophrenia.
\end{abstract}

Method Over two years, consecutive first-episode psychosis patients

presenting to a catchment area psychiatric service underwent validated clinical assessments of premorbid adjustment, illness duration, symptoms and QOL.

Results At presentation, subjects already had a diminished QOL. Although independent of gender and age at onset of psychosis, QOL was influenced by premorbid adjustment, duration of untreated psychosis and symptoms.

Conclusions Reducing the duration of untreated psychosis may have a beneficial effect on the subsequent QOL of patients presenting with schizophrenia. Firstepisode patients with a protracted duration of untreated psychosis or impaired premorbid adjustment may warrant specific treatment interventions to prevent the development of secondary handicaps.

Declaration of interest This study was funded by the Stanley Research Foundation.
Interest regarding quality of life (QOL) in those with schizophrenia has occurred in conjunction with a recognition that the prospective assessment of first-episode samples may identify determinants of outcome. To date, first-episode studies indicate that clinical outcome in schizophrenia is influenced by premorbid adjustment (Loebel et al, 1992) and the duration of untreated psychosis (DUP) (Loebel et al, 1992; Szymanski et al, 1996). Although failure to treat symptoms and side-effects adequately is associated with a poorer QOL in chronic schizophrenia (Young et al, 1998), little is known about the determinants of QOL prior to the initiation of psychiatric treatment. Consequently, we assessed the QOL of individuals presenting for the first time with schizophrenia and evaluated its clinical correlates. In the context of this study, QOL refers to a construct which incorporates aspects of an individual's well-being and role functioning and the extent to which he or she has access to resources and opportunities (Lehman, 1996).

\section{METHOD}

\section{Subjects}

Since February 1995, individuals presenting with a first episode of psychosis to the in- or out-patient departments of Cluain Mhuire Family Centre, Dublin (catchment area population 165000 ) have received a detailed clinical assessment. First episode of psychosis is defined as follows: either a first-ever presentation to any psychiatric service with a psychotic episode; or a psychotic episode receiving current treatment provided that there has been no previous treatment for psychosis or possible psychosis and that current treatment did not commence more than 30 days before referral to this service.

\section{Assessments}

At presentation, patients were assessed using the Positive and Negative Syndrome Scale (PANSS; Kay et al, 1987) and the Quality of Life Scale (QLS; Heinrichs et al, 1984), a 'disease-specific' instrument which provides an 'external assessment' of QOL based on patients' self-report and the interviewer's assessment of their current life circumstances. When patients were clinically stable, a DSM-IV diagnosis (American Psychiatric Association, 1994) was ascertained using the up-dated version of the Structured Clinical Interview for DSM-III-R - Patient Version (SCID-P; Spitzer et al, 1987).

The patients were asked to consent to a parent being interviewed using the Premorbid Social Adjustment (PSA) scale (Foerster et al, 1991), which evaluates functioning from five to eleven (PSA-1) and from 12 to 16 (PSA-2) years of age. The DUP was defined as the time between the emergence of psychotic symptoms and the initiation of treatment. It was measured in two ways: first, during the SCID interview, patients dated the onset of the first psychotic symptom; second, a family member in regular contact with the patient was interviewed, using a check-list of behaviours describing the evolution of the illness (Beiser et al, 1993).

\section{Interrater reliabilities}

Intraclass correlation coefficients (ICCs) were 0.73 for the negative syndrome subscale and in excess of 0.84 for the remaining sub-scales of the PANSS. ICCs for the QLS sub-scale scores were in excess of 0.92 , in excess of 0.88 for the PSA-1 and PSA-2 and in excess of 0.94 for the dating of the onset of prominent psychotic symptoms.

\section{Data analysis}

Data were analysed by using Student's $t$ test, the $\chi^{2}$ test or Pearson product-moment correlations (two-tailed). Information from patients regarding the emergence of psychotic symptoms was used to date their age at onset and to dichotomise them into long-term symptom (DUP of one year or greater) and short-term symptom (DUP less than one year) groups, using previously described criteria (Haas \& Sweeney, 1992). Because the development of psychosis in adolescence restricts the evaluation of premorbid adjustment, we only assessed the 
relationship between the QLS and PSA scores in individuals aged 18 years or over at presentation.

\section{RESULTS}

Over two years, 61 individuals fulfilled DSM-IV criteria for schizophrenia or schizophreniform psychosis. Although the majority required admission to hospital, nine individuals $(15 \%)$ were treated as out-patients. Five patients were referred after starting to take neuroleptics and three patients declined to complete the QLS interview. All further discussion relates to the remaining 53 individuals ( 36 male, 17 female).

The subjects' total QLS score (mean 56.4 , s.d. $=20.6$, median 57) was inversely correlated with the total PANSS score $(r=-0.34, P=0.01)$ but independent of the Global Assessment of Functioning score (GAF; American Psychiatric Association, 1987) $(r=0.09)$. Although there was no significant relationship with positive symptoms $(r=-0.02)$, the QLS score was significantly correlated with the PANSS negative $(r=-0.38, P<0.01)$ and general psychopathology $(r=-0.31, P=0.02)$ syndrome scores. However, the severity of positive symptoms was positively correlated with the general psychopathology syndrome score $(r=0.39, P<0.01)$. There was no significant difference in total QLS scores between male (mean 56.4, s.d.=20.1) and female (mean 56.5, s.d. $=22.2)$ patients $(t=-0.22$, d.f. $=51)$.

Age at onset of psychosis (mean 25.3 years, s.d. $=8.8$, range $13-45$ ) was independent of the total QLS score $(r=0.16)$ but positively correlated with the PANSS positive syndrome score $(r=0.31, P=0.02)$. However, it was independent of the PANSS negative $(r=0.001)$ and general psychopathology $(r=-0.02)$ syndrome scores.

Although the mean DUP reported by patients was 22.7 months (s.d. $=36.8$, median 6 months, range 1-240), their relatives reported a shorter DUP (mean 15.9, s.d. $=34.5$, median 3 months, range 0 240). Using the patients' dating, 23 individuals $(43.3 \%)$ were classified as having a DUP of 12 months or more (long-term symptom group). There were no significant gender differences between the longterm and short-term symptom groups $\left(\chi^{2}=2.24, \quad\right.$ d.f. $\left.=1, \quad P=0.13\right)$. However, there was a significant difference in the QLS score $(t=-2.89$, d.f. $=51, P<0.01)$ between the long-term and short-term groups, but no significant differences between these groups in the PANSS total or syndrome scores (Table 1). A stepwise multiple regression analysis indicated that a combination of a DUP of 12 months or longer $(t=-2.81, P=0.007)$ and the total PANSS score $(t=-2.41, \quad P=0.02)$ accounted for a significant proportion of the variance in the QOL of these individuals (adjusted $R^{2}=19.9 \%, \quad F=7.48$, $P=0.001$ ).

Of the 50 adults (age range 20-45 years) in this cohort, data relating to premorbid adjustment were obtained for 46 individuals. There was a significant relationship between the QLS, PSA-1 $(r=-0.32, P=0.03)$ and PSA-2 $(r=-0.39$, $P<0.01)$ scores. Although there was evidence that age at onset was related to the PSA-1 $(r=-0.28, P=0.06)$ and PSA-2 $(r=-0.41, \quad P<0.01)$ scores, premorbid adjustment was independent of the PANSS total and syndrome scores $(0.04 \leqslant r \leqslant 0.14)$. Furthermore, the longterm and short-term symptom groups did not differ in terms of the PSA-1 $(t=-0.68, \quad$ d.f. $=44) \quad$ and PSA-2 $(t=-1.23$, d.f. $=44)$ scores.

Table I Clinical comparisons between long-term and short-term symptom groups

\begin{tabular}{lccc}
\hline & Long-term $(n=23)$ & Short-term $(n=30)$ & $P$ \\
\hline Age (years) & $26.5(8.7)^{\prime}$ & $27.5(9.2)$ & 0.68 \\
Quality of Life Scale total score & $47.6(19.9)$ & $63.1(18.8)$ & $<0.01$ \\
Positive and Negative Syndrome Scale & & & \\
$\quad$ Positive syndrome & $24.1(6.1)$ & $25.2(6.2)$ & 0.50 \\
Negative syndrome & $21.1(8.2)$ & $17.6(7.8)$ & 0.10 \\
General psychopathology syndrome & $42.7(9.7)$ & $43.7(10.3)$ & 0.74 \\
Total score & $88.5(15.9)$ & $86.5(21.1)$ & 0.72 \\
\hline
\end{tabular}

I. Figures in parentheses denote s.d.

\section{DISCUSSION}

To date, there have been significant differences between studies in the approaches taken to evaluating QOL in schizophrenia, especially in relation to the use of self-rated as opposed to external assessments. It has been suggested that self-reports should be accepted at face value even if they reflect a patient's delusional beliefs (Orley et al, 1998), but other investigators have highlighted the potential for psychotic symptoms, diminished insight and neurocognitive deficits to limit the usefulness of the selfreport methodology in acutely ill populations. Furthermore, it has been suggested that these measures may not adequately reflect the effects of various interventions (Barry \& Zissi, 1997).

\section{The QLS as a measure of quality of life}

Recent reviews have produced somewhat conflicting recommendations regarding the most appropriate instrument for evaluating QOL. However, fundamental requirements of QOL measures include patient acceptability and the ability to provide data relating to psychometrics. To date, QOL instruments have been evaluated in terms of construct and convergent validity with other commonly used scales. The QLS has been reported to be acceptable in terms of these measures of validity (Lehman et al, 1993; Lehman, 1996) and to reflect prospectively the effects of pharmacological and psychosocial interventions (Rosenheck et al, 1998). Regarding patient acceptability, only three patients in this study declined to complete the QLS interview, in contrast to the relatively high rate of incomplete data reported with self-rated evaluations in in-patient samples (Kaiser et al, 1996).

Since the QLS was initially devised as a measure of the schizophrenic deficit syndrome, it could be argued that it simply documents the presence of negative symptoms. However, the concept of the deficit syndrome has evolved to require evidence that negative symptoms persist longitudinally in the absence of psychosis, depression or side-effects (Carpenter et al, 1988), and the percentage of first-episode patients who meet these criteria appears to be minimal (Mayerhoff et al, 1994). Furthermore, in the present study, negative symptom severity only accounted for $15 \%$ of the variance in the QLS score. Although this 
finding may be interpreted in terms of 'measurement redundancy', there is little point in using a QOL instrument which fails to detect the impact of symptoms specific to schizophrenia (Awad et al, 1997). In fact, the pattern of symptoms appears to be a robust predictor of subjective QOL (Kaiser et al, 1996), and the deleterious effects of negative and general psychopathological symptoms have been previously demonstrated with self-rated instruments (Packer et al, 1997; Heslegrave et al, 1997). Although core psychotic symptoms were not found to influence QOL, it may be that they have an indirect effect mediated through general psychopathological symptoms.

\section{Methodological issues in first-episode studies}

The length of untreated illness prior to presentation is likely to be an important determinant of outcome, since consecutive-admission studies indicate that the best predictor of future chronicity is past chronicity. However, there are difficulties in dating the onset of illness in first-episode samples. For example, dating the onset of the prodrome is uniquely problematic, because it is difficult to distinguish prodromal signs from the social and academic impairments which are premorbidly evident in some patients with schizophrenia.

Although our data confirm that some first-episode patients experience psychotic symptoms for a protracted period of time prior to referral, the mean DUP was greater than the median, indicating a significant effect of outliers. This reflects the difficulty in adequately defining what constitutes a first episode of schizophrenia. Furthermore, the reliability of patients' dating of psychosis onset may be questioned. However, this difficulty may be circumvented by interviewing patients when they are symptomatically stable and supplementing their report with collateral information from families (Keshevan \& Schooler, 1992). The finding that family members reported a shorter DUP than patients may be due to the fact that psychotic symptoms are subjectively experienced phenomena and are not easily perceived by others.

\section{Determinants of quality of life at presentation}

The QOL of the individuals in this study was influenced by their psychopathology, by how long they had psychosis prior to

\section{CLINICAL IMPLICATIONS}

Patients presenting with schizophrenia may have an already diminished quality of life (QOL).

- Early treatment may have a beneficial effect on QOL in first-episode schizophrenia.

First-episode patients with premorbid social maladjustment or a protracted duration of untreated psychosis (DUP) may warrant specific treatment interventions.

\section{LIMITATIONS}

- Not all first-episode patients completed the QOL assessment.

- The Quality of Life Scale attaches unequal weight to the three dimensions of QOL.

- A wide range of DUPs was reported.

STEPHEN BROWNE, MRCPsych, MARY CLARKE, MRCPsych, MAURICE GERVIN, MRCPsych, Cluain Mhuire Family Centre, Blackrock, Co. Dublin; JOHN L.WADDINGTON, DSc, Royal College of Surgeons of Ireland, Dublin; CONAL LARKIN, FRCPsych, St John of God Hospital, Stillorgan, Co. Dublin; EADBHARD O'CALLAGHAN, MRCPsych, Cluain Mhuire Family Centre, Blackrock, Co. Dublin, Republic of Ireland

Correspondence: Dr E. O'Callaghan, Stanley Foundation Research Unit, Cluain Mhuire Family Centre, Newtownpark Avenue, Blackrock, Co. Dublin, Republic of Ireland

(First received 18 May 1998, final revision 9 July 1999, accepted 9 July 1999)

referral and by their premorbid adjustment before the onset of psychotic symptoms. Since there was no difference in premorbid functioning between the long- and shortterm symptom groups, it appears that the difference in QOL between these groups is unlikely to have resulted from difficulties in the acquisition of life skills prior to the onset of psychosis. Likewise, the effect of DUP on QOL appears to be independent of the pattern of symptoms, including negative symptoms. Consequently, it may be that a protracted DUP has an inherently detrimental effect on an individual's QOL. However, it is possible that differences in the mode of illness onset or in the environmental response to the emergence of psychosis (for example, factors influencing treatment-seeking) may be an alternative explanation for this finding.

\section{REFERENCES}

American Psychiatric Association (1987) Diagnostic and Statistical Manual of Mental Disorders (3rd edn, revised) (DSM-III-R). Washington, DC: APA.
_ (1994) Diagnostic and Statistical Manual of Mental Disorders (4th edn) (DSM-IV). Washington, DC: APA

Awad, A. G., Voruganti, L. N. \& Heslegrave, R. J.

(1997) Measuring quality of life in patients with schizophrenia. Pharmacoeconomics, II, 32-47.

Barry, M. M. \& Zissi, A. (1997) Quality of life as an outcome in evaluating mental health services: a review of the empirical evidence. Social Psychiatry and Psychiatric Epidemiology, 32, 38-47.

Beiser, M., Erickson, D., Fleming, J. A., et al (1993) Establishing the onset of psychotic illness. American Journal of Psychiatry, 150, 1349-1354.

Carpenter, W. T., Heinrichs, D. W. \& Hagman, A. M. (1988) Deficit and nondeficit forms of schizophrenia: the concept. American Journal of Psychiatry, $\mathbf{1 4 5}$, 578-583.

Foerster, A., Lewis, S., Owen, M., et al (1991) Pre-morbid adjustment and personality in psychosis. Effects of sex and diagnosis. British Journal of Psychiatry, 158, $|7|-176$.

Haas, G. L. \& Sweeney, J. A. (1992) Premorbid and onset features of first-episode schizophrenia. Schizophrenia Bulletin, 18, 373-386.

Heinrichs, D. W., Hanlon, E.T. \& Carpenter, W.T. (1984) The quality of life scale: an instrument for rating the schizophrenic deficit syndrome. Schizophrenia Bulletin, 10, 388-398.

Heslegrave, R. J., Awad, A. G. \& Voruganti, L. N. (1997) The influence of neurocognitive deficits and 
symptoms on quality of life in schizophrenia. Journal of Psychiatry and Neuroscience, 22, 235-243.

Kaiser, W., Priebe, S., Barr, W., et al (1996) Profiles of subjective quality of life in schizophrenic in- and outpatient samples. Psychiatric Research, 66, 153-166.

Kay, S., Fiszbein, A. \& Opler, L. (1987) The Positive and Negative Syndrome Scale (PANSS) for schizophrenia. Schizophrenia Bulletin, 13, 261-276.

Keshevan, K. S. \& Schooler, N. R. (1992) First-episode studies in schizophrenia: criteria and characterisation. Schizophrenia Bulletin, 18, 49I-513.

Lehman, A. F. (1996) Measures of quality of life among persons with severe and persistent mental disorders. Social Psychiatry and Psychiatric Epidemiology, 3I, 78-88.

— , Postrado, L. T. \& Rachuba, L. T. (1993)

Convergent validation of quality of life assessments for persons with severe mental illnesses. Quality of Life Research, 2, 327-333.

Loebel, A., Lieberman, J. A., Alvir, J. M., et al (1992) Duration of psychosis and outcome in first-episode schizophrenia. American Journal of Psychiatry, 149, |l83-1188.

Mayerhoff, D. I., Loebel, A. D., Alvir, J. M., et al (1994) The deficit state in first-episode schizophrenia. American Journal of Psychiatry, I5I, I4I7-1422.

Orley, J., Saxena, S. \& Herrman, H. (1998) Quality of life and mental illness. Reflections from the perspective of the WHOQOL. British Journal of Psychiatry, I72, $291-293$

Packer, S., Husted, J., Cohen, S., et al (1997) Psychopathology and quality of life in schizophrenia. Journal of Psychiatry and Neuroscience, 22, 23I-234.
Rosenheck, R., Tekell, J., Peters, J., et al (1998) Does participation in psychosocial treatment augment the benefit of clozapine? Archives of General Psychiatry, 55, 618-625.

Spizter, R. L., Williams, J. B. \& Gibbon, J. (1987) Structured Clinical Interview for DSM-III-R - Patient Version (SCID-P). New York: New York State Psychiatric Institute.

Szymanski, S. R., Cannon, T. D., Gallacher, F., et al (1996) Course of treatment response in first-episode and chronic schizophrenia. American Journal of Psychiatry, 153, 519-525.

Young, A. S., Sullivan, G., Burnam, M. A., et a (1998) Measuring the quality of outpatient treatment for schizophrenia. Archives of General Psychiatry, 28, $122 \mid-1230$. 